Revta brasil. Bot., São Paulo, V.23, n.4, p.371-383, dez. 2000

\title{
Estrutura e diversidade do componente arbóreo da floresta na Estação Ecológica dos Caetetus, Gália, SP
}

\author{
GISELDA DURIGAN ${ }^{1,4}$, GERALDO ANTÔNIO DAHER CORRÊA FRANCO ${ }^{2}$, \\ MASAHIRO SAITO ${ }^{3}$ e JOÃO BATISTA BAITELLO ${ }^{2}$
}

(recebido em 11 de setembro de 1998; aceito em 17 de maio de 2000)

\begin{abstract}
Structure and diversity of the arboreal component of the forest at Caetetus Ecological Station, Gália, SP). Phytosociology of distinct tree strata in tropical forests can be an important tool to understand community dynamics. The arboreal community of an area $\left(6,000 \mathrm{~m}^{2}\right)$ of mature forest was surveyed at the Caetetus Ecological Station, Gália, São Paulo State, Brazil. This protected area has one of the last significant forest remnants in Western São Paulo State, covering an area of 2,178.84 ha in a region where coffee plantation and pasture are dominant. Although the fragment has been protected against fire and exploitation for many years, there are visible edge effects. The sampling area in this study was intended to be representative of the original primary forest, free from edge effects. The arboreal community was divided into three strata: DBH equal to or higher than $5 \mathrm{~cm}$ (upper), DBH between 1 and $5 \mathrm{~cm}$ (intermediate) and DBH below $1 \mathrm{~cm}$ (lower). In each layer, 60 permanent plots, with different sizes were installed, being: 10 x $10 \mathrm{~m}$ (upper layer); 10 x $2 \mathrm{~m}$ (intermediate layer) and 2 × $2 \mathrm{~m}$ (lower layer). The upper stratum had 1,080 ind.ha ${ }^{-1}, 62$ species (28 families), being the most important (decreasing order): Metrodorea nigra, Savia dictyocarpa, Ocotea indecora, Aspidosperma polyneuron and Trichilia catigua. The intermediate stratum had 3,525 ind.ha ${ }^{-1}, 30$ species (17 families), being the most important: Metrodorea nigra, Actinostemon concolor, Trichilia catigua, Aspidosperma polyneuron and Trichilia claussenii. The density in the lower stratum was 28,875 ind.ha $^{-1}$, from 37 species (19 families), being the most abundant, in decreasing relative density: Metrodorea nigra, Actinostemon concolor, Trichilia catigua, Aspidosperma polyneuron and Eugenia blastantha. Some of the species of the upper stratum was absent in the other strata, especially heliophytes, typical from early sucessional stages (e.g. Croton floribundus). Other species had decreasing importance values from the upper to the lower stratum (e.g. Savia dictyocarpa and Ocotea indecora). There were species whose seeds germinated but plants did not grow in the shade (e.g. Cariniana estrellensis). Finally, there was a group of species, typical from late successional stages, that had similar relative density in all strata, e.g. Metrodorea nigra and Aspidosperma polyneuron.
\end{abstract}

RESUMO - (Estrutura e diversidade do componente arbóreo da floresta na Estação Ecológica dos Caetetus, Gália, SP). O estudo fitossociológico, se realizado em diferentes estratos verticais da floresta, fornece dados de grande valia para a compreensão da dinâmica da comunidade. Efetuou-se levantamento das espécies arbóreas presentes em diferentes estratos, em área de $6.000 \mathrm{~m}^{2}$ de floresta madura, na Estação Ecológica dos Caetetus, Gália, SP. Foram utilizadas 60 parcelas de 10 x 10 m, para indivíduos com diâmetro do caule a $1,30 \mathrm{~m}$ acima do nível do solo (DAP) a partir de $5 \mathrm{~cm}$ (estrato superior); 10 × $2 \mathrm{~m}$, para indivíduos com DAP entre 1 e $5 \mathrm{~cm}$ (estrato intermediário) e 60 sub-parcelas de 2 × $2 \mathrm{~m}$, para indivíduos menores que $1 \mathrm{~cm}$ de DAP (estrato inferior). Verificou-se que a diversidade, a composição florística e a densidade relativa das espécies variam entre estratos. No estrato superior, com 1.080 indivíduos.ha $^{-1} \mathrm{e}$ área basal de $31,2 \mathrm{~m}^{2} \cdot \mathrm{ha}^{-1}$, encontraram-se 62 espécies (28 famílias), tendo sido as mais importantes, em ordem decrescente de IVI: Metrodorea nigra, Savia dictyocarpa, Ocotea indecora, Aspidosperma polyneuron e Trichilia catigua. O estrato intermediário apresentou-se com densidade de 3.525 indivíduos.ha ${ }^{-1}$, pertencentes a 30 espécies (17 famílias), sendo as mais importantes em ordem decrescente de IVI: Metrodorea nigra, Actinostemon concolor, Trichilia catigua, Aspidosperma polyneuron e Trichilia clausenii. No estrato inferior a densidade foi de 28.875 indivíduos.ha ${ }^{-1}$, pertencentes a 37 espécies (19 famílias), sendo as mais abundantes, em ordem decrescente de densidade relativa: Metrodorea nigra, Actinostemon concolor, Trichilia catigua, Aspidosperma polyneuron e Eugenia blastantha. Constatou-se que há espécies do estrato superior da floresta que não foram observadas em regeneração, especialmente heliófitas de estádios sucessionais iniciais (p. ex. Croton floribundus). Outras ocorreram com densidade relativa decrescente nos estratos inferiores (p. ex. Savia dictyocarpa e Ocotea indecora). Para outras espécies, as sementes germinaram, mas não se desenvolveram à sombra, estando ausentes no estrato intermediário (p. ex. Cariniana estrellensis). Observou-se, ainda, um grupo de espécies, de estádios sucessionais mais avançados, que apresentaram a mesma densidade relativa em todos os estratos (p. ex. Metrodorea nigra e Aspidosperma polyneuron).

Key words - Seasonal semideciduous forest, phytosociology, stratification

\section{Introdução}

1. Instituto Florestal, Estação Experimental de Assis, Caixa Postal 104, 19.800-000 Assis, SP, Brasil.

2. Instituto Florestal, Divisão de Dasonomia, Caixa Postal 1322, 01059-970 São Paulo, SP, Brasil.

3. Forestry and Forest Products Research Institute, Ibaraki, 305, Japan.

4. Autor para correspondência: giselda@femanet.com.br
A Estação Ecológica dos Caetetus localiza-se no Planalto Ocidental Paulista, região de domínio de floresta estacional semidecidual, segundo a classificação de Veloso et al. (1991). Este tipo florestal faz parte do complexo da Mata Atlântica, o mais ameaçado dos ecossistemas florestais brasileiros, apre- 
sentando atualmente menos de 9\% de sua área original (MMA 1998). Somente no Estado de São Paulo, foram destruídos, entre 1907 e 1934, cerca de $79.500 \mathrm{~km}^{2}$ desta floresta $\left(3.000 \mathrm{~km}^{2} / \mathrm{ano}\right)$, segundo Dean (1997). Dados recentes sobre o desmatamento neste estado, entre 1990 e 1995 , mostram que foram destruídos neste período $674 \mathrm{~km}^{2}$ de Mata Atlântica (MMA 1998). Na região administrativa de Marília, onde está situada a Estação Ecológica dos Caetetus, a cobertura florestal remanescente, segundo Kronka et al. (1993), é inferior a 3\%. O histórico da destruição da vegetação natural na região compreendida entre os rios Paranapanema e Peixe, onde se insere a área de estudo, foi resgatado por Brannstrom (1998), que constatou processos diferentes de ocupação nas áreas de floresta e cerrado, com papel de pequena importância relativa desempenhado pelas lavouras cafeeiras na região como um todo.

A floresta estacional semidecidual foi o tipo florestal mais rápida e extensamente devastado no Estado de São Paulo e em toda a sua área de ocorrência natural, que compreende parte dos Estados de Minas Gerais, Paraná, Santa Catarina, Rio Grande do Sul, Goiás, Mato Grosso do Sul, Bahia e Espírito Santo e de países vizinhos, como o Paraguai e a Argentina. A devastação dessas florestas ocorreu associada à expansão da fronteira agrícola, já que ocupavam os solos de maior fertilidade no Estado de São Paulo, em regiões com relevo favorável à agricultura. Dos fragmentos remanescentes, poucos têm área representativa e encontram-se preservados.

As áreas disponíveis para pesquisa sobre floresta estacional semidecidual no Estado de São Paulo são, portanto, poucas e os dados disponíveis escassos, gerados por estudos recentes, destacando-se: Cavassan et al. (1984) em Bauru, Baitello et al. (1988) e Schlittler et al. (1995) em Teodoro Sampaio, Cesar \& Leitão-Filho (1990) em Anhembi, Nicolini-Gabriel \& Pagano (1992, 1993) em Jaú, Silva-Filho \& Engel (1993) em Botucatu, Stranguetti (1996) em Paulo de Faria e Rozza (1997) em Matão.

Também escassos são os estudos que englobam estratos distintos da vegetação e que permitem a compreensão de aspectos relacionados com a dinâmica de comunidades arbóreas. Para o Estado de São Paulo, estudos abordando os estratos inferiores em região de Floresta Estacional Semidecidual foram realizados por Zickel (1995), Bernacci \& Leitão-
Filho (1996) e Rozza (1997). Estudos desta natureza em outros tipos florestais no estado foram realizados por Costa \& Mantovani (1992), Cersósimo (1993), Cardoso-Leite (1995) e Tabarelli \& Mantovani (1997).

Os métodos utilizados são muito variáveis entre os estudos, especialmente em número e área das parcelas e classes de tamanho utilizadas para estratificação da comunidade. Como exemplo, Costa \& Mantovani (1992) adotaram $15 \mathrm{~cm}$ como altura mínima das plantas, Bernacci \& Leitão-Filho (1996), $20 \mathrm{~cm}$ e Tabarelli \& Mantovani (1997), $50 \mathrm{~cm}$. Essa falta de padrão dificulta a comparação dos resultados obtidos por diferentes autores.

A identificação de estratos verticais na floresta é sempre controvertida e, em florestas tropicais, nem sempre a separação entre eles é perceptível. O conceito de estratos como camadas sobrepostas teve origem em Humboldt (1808), que caracterizava a hiléia sul americana como "uma floresta sobre a floresta". Brown (1919) descreveu florestas tropicais das Filipinas como tendo três estratos. Porém, este autor constatou que a estratificação é complexa, com indivíduos jovens do estrato superior ocorrendo nos estratos inferiores e entre os estratos. Segundo Richards (1996), o espaço entre o piso e o topo das árvores mais altas nunca é uniformemente preenchido, freqüentemente não há descontinuidade e os limites de altura entre estratos são vagos ou arbitrários. Essa opinião é compartilhada por outros autores, como Mildbraed (1922) e Hallé et al. (1978). Apesar disso, a divisão em estratos é um conceito bastante útil na análise da estrutura da floresta (Hallé et al. 1978).

Além da dificuldade de se reconhecerem estratos bem definidos, a determinação da altura das árvores em florestas é difícil e pouco precisa (Chapman \& Meyer 1949, Bruce \& Schumacher 1950). Os equipamentos para medição de árvores pressupõem a visualização, a partir de um mesmo ponto, da base e do topo da árvore, o que é, geralmente, inviável no interior das florestas tropicais. Considerando a correlação existente entre diâmetro e altura, Richards (1996) pondera que a distribuição em classes de diâmetro reflete a distribuição da altura das árvores, fornecendo, portanto, indicações sobre a estrutura vertical da floresta. 
O presente estudo foi realizado visando gerar conhecimentos sobre a estrutura e a dinâmica sucessional da Floresta Estacional Semidecidual, em local onde o ecossistema pode ser considerado não alterado pela ação antrópica e livre dos efeitos de borda decorrentes da fragmentação, sendo denominado, ao longo deste trabalho, floresta madura. Estes conhecimentos constituem-se em instrumento de grande valor para o delineamento de programas de recuperação da cobertura vegetal e para a compreensão do papel desempenhado pelas espécies na comunidade e no processo sucessional.

\section{Material e métodos}

A área de estudo localiza-se na Estação Ecológica dos Caetetus, de propriedade do Instituto Florestal de São Paulo, situada entre os paralelos $22^{\circ} 22^{\prime}$ e $22^{\circ} 26^{\prime} \mathrm{S}$ e os meridianos $49^{\circ} 40^{\prime}$ e $49^{\circ} 44^{\prime} \mathrm{W}$ (figura 1), no limite norte da bacia hidrográfica do médio Paranapanema. A Estação abrange áreas dos municípios de Gália e Alvinlândia, estando a área de amostragem localizada no município de Gália. A área de floresta correspondente à Estação foi mantida como reserva quando da ocupação agrícola da fazenda Paraíso, tendo passado a ser propriedade do Estado em 1976 e enquadrada na categoria de Estação Ecológica em 1987 (SMA 1998).

Predominam na área da Estação os Latossolos de textura média nos topos e os Podzólicos abruptos nas vertentes. No local das parcelas, o solo é Podzólico Vermelho Amarelo Profundo, textura arenosa/média (Mattos et al. 1996). O clima regional, segundo a classificação de Köppen, é Cwa, mesotérmico de inverno seco. A Estação Ecológica dos Caetetus contém uma grande diversidade de habitats, correlacionada com a vegetação, a topografia, os solos e a rede de drenagem. Este mosaico ambiental, analisado por Mattos et al. (1996), com base na interpretação de aspectos do meio físico e da vegetação, resulta em doze unidades distintas. A área nuclear da floresta corresponde à floresta com estrato arbóreo alto, classificada com base em fotointerpretação. Nesta área, foram instaladas as parcelas de amostragem, distantes cerca de $1.200 \mathrm{~m}$ da borda da mata e cerca de $800 \mathrm{~m}$ do curso d'água mais próximo, em declividades entre 3 e $5 \%$. A amostragem foi efetuada no mês de novembro, início da estação chuvosa na região.

Para compreender a estrutura da floresta, foram amostrados todos os indivíduos de espécies arbóreas com altura mínima de $10 \mathrm{~cm}$, subdivididos em três estratos. Diante das limitações mencionadas para a determinação precisa da altura dos indivíduos (Chapman \& Meyer 1949, Bruce \& Schumacher 1950) e para o reconhecimento de estratos verticais distintos (Mildbraed $1922 \mathrm{e}$ Hallé et al. 1978) e considerando-se que a distribuição das classes de diâmetro reflete a distribuição da altura (Richards 1996), adotou-se, neste estudo, a subdivisão da comunidade arbórea em três estratos, separados por classes de DAP (diâmetro do caule a $1,30 \mathrm{~m}$ acima do nível do solo): DAP $<1 \mathrm{~cm}$ (estrato inferior), $1 \mathrm{~cm} \leq \mathrm{DAP}<5 \mathrm{~cm}$ (estrato intermediário) e DAP $\geq 5 \mathrm{~cm}$ (estrato superior). A classificação dos estratos em superior, médio e

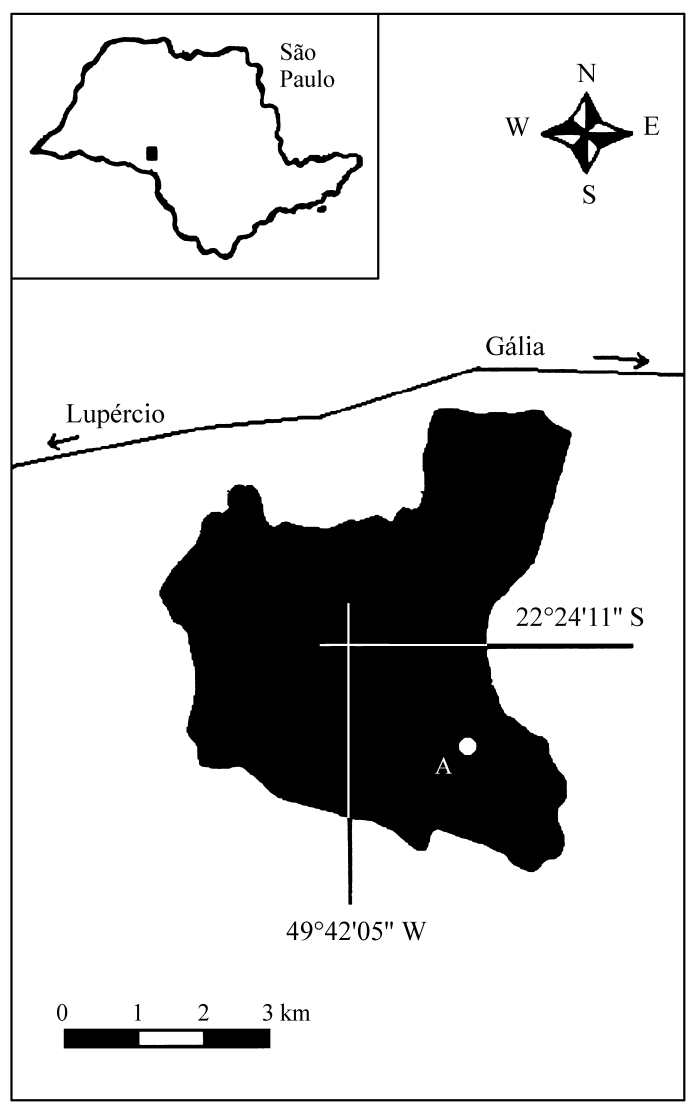

Figura 1. Localização da Estação Ecológica dos Caetetus, Gália, Alvinlândia, SP. A. área de estudo.

inferior é, portanto, relativa neste estudo, não estando diretamente relacionada com a exposição à luz ou com a separação visual de camadas do dossel. A altura dos indivíduos foi medida até $15 \mathrm{~m}$ com régua dendrométrica e, a partir desta altura, estimada visualmente.

Adotou-se para a amostragem o método de parcelas (MüllerDombois \& Ellenberg 1974), com áreas variáveis para os diferentes estratos. A área das sub-parcelas foi definida em função da densidade de indivíduos, buscando-se amostrar um número semelhante de indivíduos nos três estratos. Para chegar ao tamanho adequado da parcela para cada estrato, foram efetuadas préamostragens dos estratos inferiores, com parcelas de área crescente, até que fosse encontrada a área que fornecesse números de indivíduos por parcela que fossem semelhantes ao número encontrado no estrato superior (cerca de 10 indivíduos por parcela).

Para amostragem do estrato superior, demarcaram-se 60 parcelas contíguas de $10 \times 10 \mathrm{~m}$, onde todos os indivíduos de espécies arbóreas foram numerados com etiquetas de alumínio, sendo identificada a espécie, medidos a altura máxima e o DAP e estimado o diâmetro da copa dos indivíduos.

$\mathrm{O}$ estrato intermediário foi amostrado em 60 parcelas menores, de $10 \times 2 \mathrm{~m}$, demarcadas dentro dos limites das parcelas instaladas para o estrato superior, tendo sido numerados com 
etiquetas plásticas, identificados e medidos o DAP, a altura e o diâmetro da copa dos indivíduos.

Dentro de cada uma das 60 parcelas, demarcou-se uma parcela ainda menor, de $2 \times 2 \mathrm{~m}$, para amostragem do estrato inferior, em que os indivíduos foram identificados, sendo medida apenas sua altura.

Os cálculos fitossociológicos seguiram Müller-Dombois \& Ellenberg (1974), tendo sido efetuados com o auxílio do programa FITOPAC (Shepherd 1994) para os dois estratos superiores. Para o estrato inferior, determinou-se densidade e frequiência, assim como o índice de agregação das espécies (P), segundo Payandeh (1970), que foi determinado também para os outros estratos. Este índice possibilita a análise da estrutura horizontal das populações, segundo o grau de agregação dos indivíduos. Valores de P inferiores a 1,0 indicam distribuição regular, entre 1,0 e 1,5 correspondem a distribuição aleatória e superiores a 1,5 indicam distribuição agregada da população. Espécies com um único indivíduo amostrado não permitem análise de agregação.

Determinou-se o índice de diversidade de Shannon-Wiener com base neperiana (Ludwig \& Reynolds 1988) para cada estrato e o índice de similaridade de Sorensen (Magurran 1988), entre os estratos.

Além das medições convencionais de altura e diâmetro dos indivíduos, efetuou-se, ainda, nas 60 parcelas de $10 \times 10 \mathrm{~m}$, a contagem de lianas, considerando-se o número de caules por parcela. A contagem de lianas (lenhosas ou não) foi efetuada com o objetivo único de se conhecer a densidade de indivíduos desta forma de vida em uma floresta bem preservada e sua relação com a cobertura das copas, como subsídio a possíveis estudos de manejo. O controle das populações de lianas, sugerido por Tabanez et al. (1997), tem sido um dos aspectos mais polêmicos do manejo visando a recuperação de florestas alteradas. Há, porém, poucos dados científicos que possam trazer subsídios à tomada de decisões sobre o assunto.

Outro parâmetro não convencional avaliado foi o grau de cobertura, correspondente ao somatório da projeção das copas das árvores sobre a área de amostragem (Floyd \& Anderson 1987).

Coletou-se material botânico de todas as espécies presentes na área de estudo, para correta identificação das espécies. As exsicatas encontram-se depositadas no Herbário D. Bento Pickel, do Instituto Florestal, e duplicatas foram armazenadas na coleção botânica regional da Seção de Assis, deste Instituto.

\section{Resultados e Discussão}

As espécies amostradas estão relacionadas na tabela 1. Foram identificadas, no total, 76 espécies, pertencentes a 32 famílias.

Analisando-se os resultados mediante o índice de valor de importância das espécies (IVI), verificase que no estrato superior (tabela 2) destacaram-se, em ordem decrescente: Metrodorea nigra, especialmente pela alta densidade e frequiência, Savia dictyocarpa, Ocotea indecora e Aspidosperma polyneuron, principalmente por apresentarem indi-

Tabela 1. Espécies arbóreas presentes em diferentes estratos da floresta na Estação Ecológica dos Caetetus, Gália, SP. S. estrato superior (DAP $\geq 5 \mathrm{~cm}$ ); M. estrato intermediário $(1 \mathrm{~cm} \leq \mathrm{DAP}<5 \mathrm{~cm}$ ); I. estrato inferior (DAP $<1 \mathrm{~cm}$, altura mínima $10 \mathrm{~cm}$ ).

\begin{tabular}{|c|c|c|c|c|}
\hline Família & Espécie & $\mathrm{S}$ & $\mathrm{M}$ & $\mathrm{I}$ \\
\hline Anacardiaceae & Astronium graveolens Jacq. & $\mathrm{X}$ & & $\mathrm{X}$ \\
\hline \multirow[t]{2}{*}{ Apocynaceae } & Aspidosperma cylindrocarpon Müll. Arg. & $\mathrm{X}$ & & \\
\hline & A. polyneuron Müll. Arg. & $\mathrm{X}$ & $\mathrm{X}$ & $\mathrm{X}$ \\
\hline \multirow[t]{2}{*}{ Arecaceae } & Syagrus oleracea (Mart.) Becc. & $\mathrm{X}$ & & \\
\hline & S. romanzoffiana (Cham.) Glassm. & $\mathrm{X}$ & & $\mathrm{X}$ \\
\hline Asteraceae & Piptocarpha sellowii (Sch. Bip.) Backer & $\mathrm{X}$ & $\mathrm{X}$ & $\mathrm{X}$ \\
\hline Bignoniaceae & Zeyheria tuberculosa (Vell.) Bureau & $\mathrm{X}$ & & \\
\hline Bombacaceae & Chorisia speciosa St. -Hil. & $\mathrm{X}$ & & \\
\hline \multirow[t]{2}{*}{ Boraginaceae } & Cordia ecalyculata Vell. & $\mathrm{X}$ & & \\
\hline & Patagonula americana $\mathrm{L}$. & $\mathrm{X}$ & & \\
\hline Caesalpiniaceae & Holocalyx balansae Micheli & $\mathrm{X}$ & $\mathrm{X}$ & $\mathrm{X}$ \\
\hline Caricaceae & Jacaratia spinosa (Aubl.) A. DC. & $\mathrm{X}$ & & \\
\hline Elaeocarpaceae & Sloanea monosperma Vell. & $\mathrm{X}$ & & \\
\hline \multirow[t]{4}{*}{ Euphorbiaceae } & $\begin{array}{l}\text { Actinostemon concepcionis (Chodat \& Hassl.) Pax \& } \\
\text { K. Hoffm. }\end{array}$ & $\mathrm{X}$ & $\mathrm{X}$ & $\mathrm{X}$ \\
\hline & A. concolor (Spreng.) Müll. Arg. & $\mathrm{X}$ & $\mathrm{X}$ & $\mathrm{X}$ \\
\hline & Alchornea glandulosa Poepp. & $\mathrm{X}$ & & \\
\hline & Croton floribundus L. Spreng. & $\mathrm{X}$ & & \\
\hline
\end{tabular}


(cont.)

\begin{tabular}{|c|c|c|c|c|}
\hline Família & Espécie & $\mathrm{S}$ & M & I \\
\hline & Savia dictyocarpa Müll. Arg. & $\mathrm{X}$ & $\mathrm{X}$ & $\mathrm{X}$ \\
\hline \multirow[t]{6}{*}{ Fabaceae } & Centrolobium tomentosum Guill. ex Benth. & $\mathrm{X}$ & $\mathrm{X}$ & $\mathrm{X}$ \\
\hline & Lonchocarpus cultratus (Tul.) Malme & $\mathrm{X}$ & & $\mathrm{X}$ \\
\hline & Machaerium hirtum Raddi & $\mathrm{X}$ & & \\
\hline & Machaerium nictitans (Vell.) Benth. & $X$ & & \\
\hline & M. stipitatum Vogel & $\mathrm{X}$ & & $\mathrm{X}$ \\
\hline & Myroxylon peruiferum $\mathrm{L}$. & $\mathrm{X}$ & & \\
\hline Flacourtiaceae & Casearia sylvestris $\mathrm{Sw}$. & & & $\mathrm{X}$ \\
\hline Icacinaceae & Citronella paniculata (Mart.) Howard & & $\mathrm{X}$ & \\
\hline \multirow[t]{2}{*}{ Lauraceae } & Endlicheria paniculata (Spreng.) J.F.Macbr. & $\mathrm{X}$ & & \\
\hline & Ocotea indecora Schott ex Meissn. & $\mathrm{X}$ & $\mathrm{X}$ & $\mathrm{X}$ \\
\hline Lecythidaceae & Cariniana estrellensis (Raddi) O. Kuntze & $\mathrm{X}$ & & $\mathrm{X}$ \\
\hline Malphiguiaceae & Bunchosia pallescens Skottsb. & $\mathrm{X}$ & & \\
\hline \multirow[t]{7}{*}{ Meliaceae } & Cabralea canjerana (Vell.) Mart. & $\mathrm{X}$ & & \\
\hline & Cedrela fissilis Vell. & $\mathrm{X}$ & & \\
\hline & Guarea kunthiana A. Juss. & $\mathrm{X}$ & & \\
\hline & Trichilia catigua A. Juss. & $\mathrm{X}$ & $\mathrm{X}$ & $\mathrm{X}$ \\
\hline & T. claussenii C. DC. & $\mathrm{X}$ & $\mathrm{X}$ & $\mathrm{X}$ \\
\hline & T. elegans A. Juss. & & $\mathrm{X}$ & \\
\hline & T. pallida $\mathrm{Sw}$. & $\mathrm{X}$ & $\mathrm{X}$ & $\mathrm{X}$ \\
\hline \multirow[t]{6}{*}{ Mimosaceae } & Indeterminada & & & $\mathrm{X}$ \\
\hline & Acacia polyphyla DC. & $\mathrm{X}$ & & \\
\hline & Albizia hasslerii (Chodat) Burkart & $\mathrm{X}$ & & \\
\hline & Inga marginata Willd. & $\mathrm{X}$ & & $\mathrm{X}$ \\
\hline & I. striata Benth. & & & $\mathrm{X}$ \\
\hline & Parapiptadenia rigida (Benth.) Brenan & $\mathrm{X}$ & & \\
\hline Monimiaceae & Mollinedia widgrenii A. DC. & $\mathrm{X}$ & $\mathrm{X}$ & \\
\hline Moraceae & Ficus insipida Willd. & $\mathrm{X}$ & & \\
\hline \multirow[t]{8}{*}{ Myrtaceae } & Campomanesia rhombea O. Berg. & $\mathrm{X}$ & $\mathrm{X}$ & $\mathrm{X}$ \\
\hline & C. xanthocarpa O. Berg. & $\mathrm{X}$ & & $\mathrm{X}$ \\
\hline & Eugenia blastantha (O. Berg.) D.Legrand & & & $\mathrm{X}$ \\
\hline & E. moraviana O. Berg. & $\mathrm{X}$ & $\mathrm{X}$ & $\mathrm{X}$ \\
\hline & Myrciaria sp.1 & $\mathrm{X}$ & & $\mathrm{X}$ \\
\hline & Myrciaria sp.2 & $\mathrm{X}$ & & $\mathrm{X}$ \\
\hline & Neomytranthes glomerata (D.Legrand) D.Legrand & $\mathrm{X}$ & & \\
\hline & Eugenia sp. & & & $\mathrm{X}$ \\
\hline Nyctaginaceae & Pisonia ambigua Heimerl & $\mathrm{X}$ & & \\
\hline \multirow[t]{2}{*}{ Phytolaccaceae } & Gallesia integrifolia (Spreng.) Harms. & $\mathrm{X}$ & & \\
\hline & Seguieria floribunda Benth. & & $\mathrm{X}$ & \\
\hline
\end{tabular}


(cont.)

\begin{tabular}{|c|c|c|c|c|}
\hline Família & Espécie & $\mathrm{S}$ & $\mathrm{M}$ & $\mathrm{I}$ \\
\hline Polygonaceae & Ruprechtia laxiflora Meissn. & & $\mathrm{X}$ & \\
\hline Rhamnaceae & Rhamnidium elaeocarpum Reissek & $\mathrm{X}$ & & $\mathrm{X}$ \\
\hline \multirow[t]{3}{*}{ Rubiaceae } & Coutarea hexandra (Jacq.) K. Schum. & & $\mathrm{X}$ & \\
\hline & Ixora venulosa Benth. & $\mathrm{X}$ & & \\
\hline & Randia armata (Sw.) DC. & & & $\mathrm{X}$ \\
\hline \multirow[t]{8}{*}{ Rutaceae } & Balfourodendron riedelianum (Engl.) Engl. & $\mathrm{X}$ & $\mathrm{X}$ & \\
\hline & Esenbeckia febrifuga (A. St.-Hil) A. Juss. ex Mart. & & $\mathrm{X}$ & $\mathrm{X}$ \\
\hline & E. grandiflora Mart. & $\mathrm{X}$ & $\mathrm{X}$ & \\
\hline & Metrodorea nigra A. St.-Hil. & $\mathrm{X}$ & $\mathrm{X}$ & $\mathrm{X}$ \\
\hline & Pilocarpus pauciflorus A. St.-Hil & $\mathrm{X}$ & & \\
\hline & P. pennatifolius Lem. & $\mathrm{X}$ & $\mathrm{X}$ & $\mathrm{X}$ \\
\hline & Zanthoxylum hiemale A. St.-Hil & $\mathrm{X}$ & & \\
\hline & Z. riedelianum Engl. & $\mathrm{X}$ & $\mathrm{X}$ & $\mathrm{X}$ \\
\hline \multirow[t]{3}{*}{ Sapindaceae } & Cupania vernalis Cambess. & & $\mathrm{X}$ & $\mathrm{X}$ \\
\hline & C. zanthoxyloides Cambess. & & $\mathrm{X}$ & \\
\hline & Diatenopteryx sorbifolia Radlk. & $\mathrm{X}$ & & $\mathrm{X}$ \\
\hline Sapotaceae & Chrysophyllum gonocarpum (Mart. \& Eichler) Engl. & $\mathrm{X}$ & $\mathrm{X}$ & $\mathrm{X}$ \\
\hline Simaroubaceae & Picramnia warmingiana Engl. & $\mathrm{X}$ & & $\mathrm{X}$ \\
\hline \multirow[t]{3}{*}{ Solanaceae } & Cestrum calycinum Willd. & & $\mathrm{X}$ & \\
\hline & Solanum argenteum Dunal & $\mathrm{X}$ & $\mathrm{X}$ & $\mathrm{X}$ \\
\hline & S. inaequale Vell. & $\mathrm{X}$ & & \\
\hline
\end{tabular}

víduos de grande porte (alta dominância). A altura média das árvores neste estrato foi de 9,6 m e a altura máxima $32,0 \mathrm{~m}$. O dossel foi dominado por Metrodorea nigra, cujos indivíduos, embora não tenham ultrapassado $12,0 \mathrm{~m}$ de altura, corresponderam a $47 \%$ do total. No estrato intermediário (tabela 3), as espécies que apresentaram os maiores valores de IVI foram, em ordem decrescente: Metrodorea nigra, Actinostemon concolor, Trichilia catigua e Aspidosperma polyneuron. A altura máxima das árvores neste estrato foi de $8,1 \mathrm{~m} \mathrm{e}$ a média 3,6 m. No estrato inferior (tabela 4), as espécies foram classificadas em ordem decrescente de densidade relativa, tendo se destacado: Metrodorea nigra, Actinostemon concolor, Trichilia catigua e Aspidosperma polyneuron. A altura máxima dos indivíduos neste estrato foi de 2,3 m e a média $0,6 \mathrm{~m}$.

Os índices de valor de cobertura (IVC) seguiram praticamente a mesma ordem do IVI para as espécies mais abundantes. Para espécies com pequeno número de indivíduos, e de grande porte, ocorreram alterações maiores, como é o caso de Cariniana estrellensis, com a 19a posição em ordem de importância e a 13a posição em ordem de cobertura ou Cedrela fissilis, que ocupou a 10aㅡ posição em ordem de importância e a sexta posição em ordem de cobertura, ambas no estrato superior (tabela 2).

Vinte e nove espécies (38\% do total) não foram observadas em regeneração na área de estudo, especialmente heliófitas, como Croton floribundus, Cedrela fissilis, Chorisia speciosa, Gallesia integrifolia e Alchornea glandulosa, que não foram amostradas nos estratos inferiores.

Um segundo grupo reuniu 11 espécies (14\% do total), cujas sementes germinaram, mas aparentemente os indivíduos não se desenvolveram à sombra, uma vez que foram amostrados nos estratos superior e inferior, mas não o foram no estrato intermediário, incluindo: Cariniana estrellensis, Lonchocarpus 
Tabela 2. Espécies presentes no estrato superior da floresta ((DAP $\geq 5 \mathrm{~cm})$, em ordem decrescente de IVI (índice de valor de importância), em floresta madura na Estação Ecológica dos Caetetus, Gália, SP. DA. densidade absoluta (ind.ha ${ }^{-1}$ ); DR. densidade relativa (\%), FA. frequiência absoluta (\%); FR. freqüência relativa (\%); DoA. dominância absoluta (área basal, $\mathrm{em} \mathrm{m}^{2}$.ha ${ }^{-1}$ ); DoR. dominância relativa (\%); IVC. índice de valor de cobertura; P. índice de agregação de Payandeh.

\begin{tabular}{|c|c|c|c|c|c|c|c|c|c|}
\hline Espécie & $\mathrm{DA}$ & $\mathrm{DR}$ & FA & FR & DoA & DoR & IVI & IVC & $\mathrm{P}$ \\
\hline Metrodorea nigra & 502 & 46,8 & 98,3 & 18,3 & 3,05 & 9,8 & 74,8 & 56,6 & 1,45 \\
\hline Savia dictyocarpa & 132 & 12,2 & 45,0 & 8,4 & 5,48 & 17,6 & 38,1 & 29,8 & 2,66 \\
\hline Ocotea indecora & 62 & 5,7 & 46,7 & 8,7 & 3,27 & 10,5 & 24,9 & 16,2 & 1,11 \\
\hline Aspidosperma polyneuron & 38 & 3,6 & 30,0 & 5,6 & 3,27 & 11,5 & 20,6 & 15,0 & 1,16 \\
\hline Trichilia catigua & 45 & 4,2 & 38,3 & 7,1 & 0,31 & 1,0 & 12,3 & 5,2 & 0,94 \\
\hline Gallesia integrifolia & 3 & 0,3 & 3,3 & 0,6 & 2,18 & 7,0 & 7,9 & 7,3 & 0,98 \\
\hline Syagrus romanzoffiana & 20 & 1,9 & 18,3 & 3,4 & 0,72 & 2,3 & 7,6 & 4,2 & 0,98 \\
\hline Croton floribundus & 18 & 1,7 & 11,7 & 2,2 & 0,86 & 2,8 & 6,6 & 4,4 & 2,12 \\
\hline Chrysophyllum gonocarpum & 17 & 1,5 & 16,7 & 3,1 & 0,47 & 1,5 & 6,2 & 3,1 & 0,85 \\
\hline Cedrela fissilis & 3 & 0,3 & 3,3 & 0,6 & 1,53 & 4,9 & 5,9 & 5,2 & 0,98 \\
\hline Parapiptadenia rigida & 7 & 0,6 & 6,7 & 1,2 & 1,10 & 3,5 & 5,4 & 4,1 & 0,95 \\
\hline Trichilia pallida & 17 & 1,5 & 16,7 & 3,1 & 0,11 & 0,4 & 5,0 & 1,9 & 0,85 \\
\hline Cabralea canjerana & 8 & 0,3 & 8,3 & 1,6 & 0,80 & 2,6 & 4,9 & 3,4 & 0,93 \\
\hline Centrolobium tomentosum & 7 & 0,6 & 6,7 & 1,2 & 0,86 & 2,8 & 4,6 & 3,4 & 0,95 \\
\hline Zanthoxyllum riedelianum & 15 & 1,4 & 13,3 & 2,5 & 0,07 & 0,2 & 4,1 & 1,6 & 1,09 \\
\hline Trichilia claussenii & 12 & 1,1 & 11,7 & 2,2 & 0,24 & 0,8 & 4,0 & 1,9 & 0,78 \\
\hline Mollinedia widgrenii & 12 & 1,1 & 11,7 & 2,2 & 0,23 & 0,7 & 4,0 & 1,8 & 0,90 \\
\hline Sloanea monosperma & 12 & 1,1 & 11,7 & 2,2 & 0,18 & 0,6 & 3,8 & 1,7 & 0,90 \\
\hline Cariniana estrellensis & 2 & 0,2 & 1,7 & 0,3 & 0,96 & 3,1 & 3,6 & 3,3 & - \\
\hline Patagonula americana & 8 & 0,8 & 6,7 & 1,2 & 0,48 & 1,5 & 3,6 & 2,3 & 0,93 \\
\hline Astronium graveolens & 10 & 0,9 & 10,0 & 1,9 & 0,18 & 0,6 & 3,4 & 1,5 & 0,92 \\
\hline Balfourodendron riedelianum & 8 & 0,8 & 8,3 & 1,6 & 0,31 & 1,0 & 3,3 & 1,8 & 0,93 \\
\hline Alchornea glandulosa & 7 & 0,6 & 6,7 & 1,2 & 0,41 & 1,3 & 3,2 & 1,9 & 0,95 \\
\hline Eugenia moraviana & 10 & 0,9 & 10,0 & 1,9 & 0,11 & 0,3 & 3,1 & 1,3 & 0,92 \\
\hline Albizia hasslerii & 3 & 0,3 & 3,3 & 0,6 & 0,41 & 1,3 & 2,3 & 1,6 & 0,98 \\
\hline Rhamnidium elaeocarpum & 5 & 0,5 & 5,0 & 0,9 & 0,24 & 0,8 & 2,2 & 1,2 & 0,97 \\
\hline Jacaratia spinosa & 3 & 0,3 & 3,3 & 0,6 & 0,34 & 1,1 & 2,0 & 1,4 & 0,98 \\
\hline Machaerium hirtum & 3 & 0,3 & 3,3 & 0,6 & 0,32 & 1,0 & 2,0 & 1,3 & 0,98 \\
\hline M. stipitatum & 5 & 0,5 & 5,0 & 0,9 & 0,15 & 0,5 & 1,9 & 0,9 & 0,97 \\
\hline Cordia ecalyculata & 7 & 0,6 & 5,0 & 0,9 & 0,06 & 0,2 & 1,7 & 0,8 & 1,46 \\
\hline Syagrus oleracea & 5 & 0,5 & 5,0 & 0,9 & 0,10 & 0,3 & 1,7 & 0,8 & 0,97 \\
\hline Chorisia speciosa & 2 & 0,2 & 1,7 & 0,3 & 0,36 & 1,2 & 1,6 & 1,3 & - \\
\hline Actinostemon concolor & 5 & 0,5 & 5,0 & 0,9 & 0,02 & 0,1 & 1,5 & 0,5 & 0,97 \\
\hline Lonchocarpus cultratus & 2 & 0,2 & 1,7 & 0,3 & 0,29 & 0,9 & 1,4 & 1,1 & - \\
\hline Campomanesia rhombea & 3 & 0,3 & 3,3 & 0,6 & 0,13 & 0,4 & 1,3 & 0,7 & 0,98 \\
\hline Diatenopteryx sorbifolia & 2 & 0,2 & 1,7 & 0,3 & 0,26 & 0,8 & 1,3 & 1,0 & - \\
\hline Guarea kunthiana & 3 & 0,2 & 3,3 & 0,6 & 0,11 & 0,4 & 1,3 & 0,7 & 0,98 \\
\hline Acacia polyphylla & 2 & 0,2 & 1,7 & 0,3 & 0,25 & 0,8 & 1,3 & 1,0 & - \\
\hline
\end{tabular}


(cont.)

\begin{tabular}{|c|c|c|c|c|c|c|c|c|c|}
\hline Espécie & $\mathrm{DA}$ & DR & FA & FR & DoA & DoR & IVI & IVC & $\mathrm{P}$ \\
\hline Zeyheria tuberculosa & 3 & 0,2 & 3,3 & 0,6 & 0,07 & 0,2 & 1,2 & 0,5 & 0,98 \\
\hline Solanum argenteum & 5 & 0,2 & 3,3 & 0,6 & 0,02 & 0,1 & 1,1 & 0,5 & 1,64 \\
\hline Campomanesia xanthocarpa & 3 & 0,2 & 3,3 & 0,6 & 0,05 & 0,2 & 1,1 & 0,5 & 0,98 \\
\hline Actinostemon concepcionis & 3 & 0,3 & 3,3 & 0,6 & 0,01 & 0,0 & 1,0 & 0,3 & 0,98 \\
\hline Esenbeckia grandiflora & 3 & 0,3 & 3,3 & 0,6 & 0,01 & 0,03 & 1,0 & 0,3 & 0,98 \\
\hline Pisonia ambigua & 2 & 0,2 & 1,7 & 0,3 & 0,13 & 0,4 & 0,9 & 0,6 & - \\
\hline Piptocarpha sellowii & 2 & 0,5 & 1,7 & 0,3 & 0,01 & 0,1 & 0,8 & 0,5 & - \\
\hline Machaerium nictitans & 2 & 0,2 & 1,7 & 0,3 & 0,11 & 0,3 & 0,8 & 0,5 & - \\
\hline Solanum inaequale & 2 & 0,2 & 1,7 & 0,3 & 0,05 & 0,2 & 0,6 & 0,3 & - \\
\hline Bunchosia pallescens & 2 & 0,2 & 1,7 & 0,3 & 0,03 & 0,1 & 0,6 & 0,3 & - \\
\hline Myrciaria sp.1 & 2 & 0,2 & 1,7 & 0,3 & 0,03 & 0,1 & 0,6 & 0,3 & - \\
\hline Myroxylon peruiferum & 2 & 0,2 & 1,7 & 0,3 & 0,03 & 0,1 & 0,6 & 0,2 & - \\
\hline Neomytranthes glomerata & 2 & 0,2 & 1,7 & 0,3 & 0,02 & 0,1 & 0,5 & 0,2 & - \\
\hline Holocalyx balansae & 2 & 0,2 & 1,7 & 0,3 & 0,01 & 0,0 & 0,5 & 0,2 & - \\
\hline Ficus insipida & 2 & 0,2 & 1,7 & 0,3 & 0,01 & 0,0 & 0,5 & 0,2 & - \\
\hline Aspidosperma cylindrocarpon & 2 & 0,2 & 1,7 & 0,3 & 0,01 & 0,0 & 0,5 & 0,2 & - \\
\hline Pilocarpus pauciflorus & 2 & 0,2 & 1,7 & 0,3 & 0,01 & 0,0 & 0,5 & 0,2 & - \\
\hline Inga marginata & 2 & 0,2 & 1,7 & 0,3 & 0,00 & 0,0 & 0,5 & 0,2 & - \\
\hline Picramnia warmingiana & 2 & 0,2 & 1,7 & 0,3 & 0,00 & 0,0 & 0,5 & 0,2 & - \\
\hline Zanthoxyllum hiemale & 2 & 0,2 & 1,7 & 0,3 & 0,00 & 0,0 & 0,5 & 0,2 & - \\
\hline Pilocarpus pennatifolius & 2 & 0,2 & 1,7 & 0,3 & 0,00 & 0,0 & 0,5 & 0,2 & - \\
\hline Myrciaria sp.2 & 2 & 0,2 & 1,7 & 0,3 & 0,00 & 0,0 & 0,5 & 0,2 & - \\
\hline Endlicheria paniculata & 2 & 0,2 & 1,7 & 0,3 & 0,00 & 0,0 & 0,5 & 0,2 & - \\
\hline Ixora venulosa & 2 & 0,2 & 1,7 & 0,3 & 0,00 & 0,0 & 0,5 & 0,2 & - \\
\hline
\end{tabular}

cultratus, Astronium graveolens e Syagrus romanzoffiana.

Quinze espécies (20\% do total) ocorreram apenas nos estratos inferiores, sendo quase todas elas umbrófilas de pequeno porte, como: Actinostemon concepcionis, A. concolor, Pilocarpus pennatifolius, P. pauciflorus e Randia armata.

Dezoito espécies (24\% do total) tiveram indivíduos amostrados nos três estratos, algumas com densidade relativa maior no estrato superior (Savia dictyocarpa, Ocotea indecora e Centrolobium tomentosum), outras com a mesma densidade relativa nos três estratos (Metrodorea nigra, Aspidosperma polyneuron e Trichilia catigua).

Três espécies (4\% do total) foram observadas nos estratos superior e intermediário: Mollinedia widgrenii, Balfourodendron riedelianum e Esenbeckia grandiflora.

Na tabela 5 são apresentados parâmetros fitossociológicos para os três estratos. A riqueza de espécies foi consideravelmente maior no estrato superior (62 espécies, 28 famílias), em relação ao estrato inferior (37 espécies, 19 famílias) e ao estrato intermediário (30 espécies, 17 famílias). A porcentagem de espécies representadas por um único indivíduo na área amostral foi também bastante variável entre estratos, correspondendo a 37\% das espécies no estrato superior, $57 \%$ no estrato intermediário e $22 \%$ no estrato inferior.

A diversidade florística, calculada segundo o índice de Shannon-Wiener (base neperiana), foi maior no estrato superior $\left(H^{\prime}=2,41\right)$, seguido do 
Tabela 3. Espécies presentes no estrato intermediário $(1 \mathrm{~cm} \leq \mathrm{DAP}<5 \mathrm{~cm})$ de floresta madura na Estação Ecológica dos Caetetus, Gália, SP, em ordem decrescente de IVI (índice de valor de importância). DA. densidade absoluta (indivíduos.ha ${ }^{-1}$ ); DR. densidade relativa (\%); FA. freqüência relativa (\%); FR. frequiência relativa (\%); DoA. dominância absoluta (área basal, em $\mathrm{m}^{2} \cdot \mathrm{ha}^{-1}$ ); DoR. dominância relativa (\%); IVC. índice de valor de cobertura; P. índice de agregação de Payandeh.

\begin{tabular}{|c|c|c|c|c|c|c|c|c|c|}
\hline Espécie & DA & $\mathrm{DR}$ & FA & FR & DoA & DoR & IVI & IVC & $\mathrm{P}$ \\
\hline Metrodorea nigra & 1797 & 51,3 & 93,2 & 29,9 & 1,1725 & 58,3 & 139,5 & 109,6 & 1,57 \\
\hline Actinostemon concolor & 551 & 15,7 & 50,9 & 16,3 & 0,2604 & 13,7 & 45,7 & 29,4 & 2,19 \\
\hline Trichilia catigua & 228 & 6,5 & 30,5 & 9,8 & 0,1306 & 6,5 & 22,8 & 13,0 & 1,39 \\
\hline Aspidosperma polyneuron & 220 & 6,3 & 30,5 & 9,8 & 0,0566 & 2,8 & 18,9 & 9,1 & 1,91 \\
\hline Trichilia claussenii & 178 & 5,1 & 23,7 & 7,6 & 0,1146 & 5,7 & 18,4 & 10,8 & 1,19 \\
\hline Savia dictyocarpa & 186 & 5,3 & 22,0 & 7,1 & 0,0698 & 3,5 & 15,9 & 8,8 & 2,44 \\
\hline Zanthoxyllum riedelianum & 59 & 1,7 & 8,5 & 2,7 & 0,0418 & 2,1 & 6,5 & 3,8 & 1,48 \\
\hline Ocotea indecora & 34 & 1,0 & 3,4 & 1,1 & 0,0266 & 1,3 & 3,4 & 2,3 & 2,48 \\
\hline Pilocarpus pennatifolius & 34 & 1,0 & 5,1 & 1,6 & 0,0050 & 0,3 & 2,9 & 1,3 & 1,46 \\
\hline Solanum argenteum & 17 & 0,5 & 3,4 & 1,1 & 0,0120 & 0,6 & 2,2 & 1,1 & 1,97 \\
\hline Centrolobium tomentosum & 17 & 0,5 & 3,4 & 1,1 & 0,0138 & 0,7 & 2,3 & 1,2 & 0,98 \\
\hline Holocalyx balansae & 17 & 0,5 & 3,4 & 1,1 & 0,0114 & 0,6 & 2,2 & 1,1 & 0,98 \\
\hline Piptocarpha sellowii & 17 & 0,5 & 3,4 & 1,1 & 0,0108 & 0,5 & 2,1 & 1,0 & 0,98 \\
\hline Actinostemon concepcionis & 9 & 0,2 & 1,7 & 0,5 & 0,0040 & 0,2 & 0,9 & 0,4 & 0,98 \\
\hline Seguieria floribunda & 9 & 0,2 & 1,7 & 0,5 & 0,0134 & 0,7 & 1,4 & 0,9 & - \\
\hline Cestrum calycinum & 9 & 0,2 & 1,7 & 0,5 & 0,0094 & 0,5 & 1,2 & 0,7 & - \\
\hline Esenbeckia grandiflora & 9 & 0,2 & 1,7 & 0,5 & 0,0070 & 0,4 & 1,1 & 0,6 & - \\
\hline Trichilia palida & 9 & 0,2 & 1,7 & 0,5 & 0,0056 & 0,3 & 1,0 & 0,5 & - \\
\hline Chrysophyllum gonocarpum & 9 & 0,2 & 1,7 & 0,5 & 0,0052 & 0,3 & 1,0 & 0,5 & - \\
\hline Coutarea hexandra & 9 & 0,2 & 1,7 & 0,5 & 0,0042 & 0,2 & 0,9 & 0,4 & - \\
\hline Esenbeckia febrifuga & 9 & 0,2 & 1,7 & 0,5 & 0,0042 & 0,2 & 0,9 & 0,4 & - \\
\hline Cupania vernalis & 9 & 0,2 & 1,7 & 0,5 & 0,0034 & 0,2 & 0,9 & 0,4 & - \\
\hline Balfourodendron riedelianum & 9 & 0,2 & 1,7 & 0,5 & 0,0020 & 0,1 & 0,8 & 0,3 & - \\
\hline Ruprechtia laxiflora & 9 & 0,2 & 1,7 & 0,5 & 0,0018 & 0,1 & 0,8 & 0,3 & - \\
\hline Mollinedia widgrenii & 9 & 0,2 & 1,7 & 0,5 & 0,0016 & 0,1 & 0,8 & 0,3 & - \\
\hline Cupania zanthoxyloides & 9 & 0,2 & 1,7 & 0,5 & 0,0014 & 0,1 & 0,8 & 0,3 & - \\
\hline Eugenia moraviana & 9 & 0,2 & 1,7 & 0,5 & 0,0012 & 0,1 & 0,8 & 0,3 & - \\
\hline Campomanesia rhombea & 9 & 0,2 & 1,7 & 0,5 & 0,0010 & 0,1 & 0,8 & 0,3 & - \\
\hline Citronella paniculata & 9 & 0,2 & 1,7 & 0,5 & 0,0008 & 0,0 & 0,7 & 0,2 & - \\
\hline Trichilia elegans & 9 & 0,2 & 1,7 & 0,5 & 0,0008 & 0,0 & 0,7 & 0,2 & - \\
\hline
\end{tabular}

estrato inferior $\left(\mathrm{H}^{\prime}=2,01\right)$ e intermediário $\left(\mathrm{H}^{\prime}=\right.$ 1,83). Estes índices de diversidade são baixos, se comparados a outras formações florestais do Estado de São Paulo. Cavassan et al. (1984), estudando o estrato arbóreo da floresta em Bauru, encontraram H' = 3,50; Schlittler et al. (1995) obtiveram H' = 4,02 em Teodoro Sampaio, Silva-
Filho \& Engel (1993) obtiveram 3,06 em Botucatu, Nicolini-Gabriel\&Pagano(1993), encontraram,em Jaú, H' = 4,06 e Rozza (1997) obteve H' = 3,24 em Matão.

Para o estrato inferior (DAP $<15 \mathrm{~cm}$ e altura $>1,30 \mathrm{~m}$ ), Rozza (1997) obteve, em Matão, $H^{\prime}=1,59$, resultado semelhante aos obtidos neste 
estudo. Em São Roque, região de floresta ombrófila densa, o estrato inferior (DAP $<5 \mathrm{~cm}$ ) apresentou diversidade alta nos três locais estudados por Cardoso-Leite (1995): H' igual a 3,18, 3,29 e 2,94, apontando para uma dinâmica sucessional distinta da floresta estacional semidecidual.

A densidade de indivíduos de espécies arbóreas com DAP a partir de $5 \mathrm{~cm}$ foi de 1.080 indivíduos.ha ${ }^{-1}$,

Tabela 4. Espécies presentes no estrato inferior da floresta (DAP $<1 \mathrm{~cm}$ ), na Estação Ecológica dos Caetetus, Gália, SP, em ordem decrescente de densidade relativa. DA. densidade absoluta (indivíduos.ha $\left.{ }^{-1}\right)$; FA. frequiência absoluta (\%); FR. freqüência relativa $(\%)$; DR. densidade relativa (\%); P. índice de agregação de Payandeh.

\begin{tabular}{|c|c|c|c|c|c|}
\hline Espécie & FA & FR & DA & $\mathrm{DR}$ & $\mathrm{P}$ \\
\hline Metrodorea nigra & 85,0 & 16,1 & 10043 & 34,8 & 3,52 \\
\hline Actinostemon concolor & 58,3 & 11,1 & 3250 & 11,3 & 3,22 \\
\hline Trichilia catigua & 45,0 & 8,5 & 1959 & 6,8 & 3,54 \\
\hline Aspidosperma polyneuron & 33,3 & 6,3 & 1667 & 5,8 & 2,34 \\
\hline Eugenia blastantha & 21,7 & 4,1 & 1584 & 5,5 & 1,45 \\
\hline Cariniana estrellensis & 33,3 & 6,3 & 1500 & 5,2 & 2,05 \\
\hline Savia dictyocarpa & 21,7 & 4,1 & 1417 & 4,9 & 3,97 \\
\hline Actinostemon concepcionis & 18,3 & 3,5 & 1083 & 3,7 & 2,61 \\
\hline Eugenia moraviana & 25,0 & 4,7 & 917 & 3,1 & 1,57 \\
\hline Trichilia clausenii & 18,3 & 3,5 & 583 & 2,0 & 1,36 \\
\hline Lonchocarpus cultratus & 10,0 & 1,9 & 458 & 1,6 & 2,86 \\
\hline Inga striata & 15,0 & 2,8 & 417 & 1,4 & 1,05 \\
\hline Machaerium stipitatum & 11,7 & 2,2 & 375 & 1,3 & 1,32 \\
\hline Pilocarpus pennatifolius & 10,0 & 1,9 & 375 & 1,3 & 1,54 \\
\hline Esenbeckia febrifuga & 13,3 & 2,5 & 333 & 1,2 & 0,88 \\
\hline Campomanesia xanthocarpa & 8,3 & 1,6 & 292 & 1,0 & 1,19 \\
\hline Mimosaceae & 11,7 & 2,2 & 292 & 1,0 & 0,90 \\
\hline Syagrus romanzoffiana & 10,0 & 1,9 & 292 & 1,0 & 1,19 \\
\hline Campomanesia rhombea & 8,3 & 1,6 & 208 & 0,7 & 0,93 \\
\hline Centrolobium tomentosum & 6,7 & 1,3 & 208 & 0,7 & 1,34 \\
\hline Diatenopteryx sorbifolia & 8,3 & 1,6 & 208 & 0,7 & 0,93 \\
\hline Inga marginata & 8,3 & 1,6 & 208 & 0,7 & 0,63 \\
\hline Zanthoxylum riedelianum & 6,7 & 1,3 & 208 & 0,7 & 1,34 \\
\hline Ocotea indecora & 6,7 & 1,3 & 167 & 0,6 & 0,95 \\
\hline Eugenia sp. & 5,0 & 0,9 & 125 & 0,4 & 1,64 \\
\hline Randia armata & 3,3 & 0,6 & 125 & 0,4 & 0,97 \\
\hline Astronium graveolens & 3,3 & 0,6 & 83 & 0,3 & 0,50 \\
\hline Chrysophyllum gonocarpum & 3,3 & 0,6 & 83 & 0,3 & 0,98 \\
\hline Trichilia palida & 3,3 & 0,6 & 83 & 0,3 & 0,98 \\
\hline Casearia sylvestris & 1,7 & 0,3 & 42 & 0,1 & - \\
\hline Cupania vernalis & 1,7 & 0,3 & 42 & 0,1 & - \\
\hline Holocalyx balansae & 1,7 & 0,3 & 42 & 0,1 & - \\
\hline Myrciaria sp.2 & 1,7 & 0,3 & 42 & 0,1 & - \\
\hline Picramnia warmingiana & 1,7 & 0,3 & 42 & 0,1 & - \\
\hline Piptocarpha sellowii & 1,7 & 0,3 & 42 & 0,1 & - \\
\hline Rhamnidium elaeocarpum & 1,7 & 0,3 & 42 & 0,1 & - \\
\hline Solanum argenteum & 1,7 & 0,3 & 42 & 0,1 & - \\
\hline
\end{tabular}


Tabela 5. Parâmetros fitossociológicos dos três estratos da floresta na Estação Ecológica dos Caetetus, Gália, SP. S. estrato superior $(\mathrm{DAP} \geq 5 \mathrm{~cm})$; M. estrato intermediário $(1 \mathrm{~cm} \leq \mathrm{DAP}<5 \mathrm{~cm})$; I. estrato inferior (DAP $<1 \mathrm{~cm}$, altura mínima $10 \mathrm{~cm})$.

\begin{tabular}{|c|c|c|c|}
\hline Parâmetro & $\mathrm{S}$ & M & $\mathrm{I}$ \\
\hline Número de espécies & 62 & 30 & 37 \\
\hline Número de famílias & 28 & 17 & 19 \\
\hline H' (Índice de Diversidade de Shannon-Wiener) & 2,41 & 1,83 & 2,01 \\
\hline Densidade (ind..ha ${ }^{-1}$ ) & 1.080 & 3.525 & 28.875 \\
\hline Área Basal $\left(\mathrm{m}^{2} \cdot \mathrm{ha}^{-1}\right)$ & 31 & 2 & - \\
\hline Cobertura das copas $(\%)$ & 179 & 59 & - \\
\hline Espécies com um único indivíduo amostrado (\%) & 37 & 57 & 22 \\
\hline
\end{tabular}

aumentando para 3.525 indivíduos. $\mathrm{ha}^{-1}$ entre $1 \mathrm{e} 5 \mathrm{~cm}$ de DAP e 28.875 indivíduos.ha ${ }^{-1}$ para plantas com DAP menor que $1 \mathrm{~cm}$ (altura mínima de $10 \mathrm{~cm}$ ). A área basal total da floresta foi de $33 \mathrm{~m}^{2} \cdot \mathrm{ha}^{-1}$, sendo $31 \mathrm{~m}^{2} \cdot \mathrm{ha}^{-1}$ correspondentes ao estrato superior e $2 \mathrm{~m}^{2} \cdot \mathrm{ha}^{-1}$ ao estrato intermediário.

Analisando-se os três estratos amostrados quanto à similaridade florística entre eles, encontram-se os seguintes resultados: $44 \%$ entre os estratos superior e intermediário, $59 \%$ entre os estratos superior e inferior e $60 \%$ entre o estrato intermediário e o estrato inferior. Os diferentes valores de similaridade obtidos mais foram decorrentes das diferenças no número de espécies do que da presença de espécies diferentes entre estratos.

Foram observadas, também, entre os estratos, diferenças quanto ao padrão de distribuição horizontal das populações de algumas espécies, analisado com base no índice de agregação de Payandeh 1970 (tabelas 2, 3, 4). De modo geral, poucas espécies apresentaram distribuição agregada, correspondendo a $3 \%$ das espécies presentes no estrato superior, $20 \%$ no estrato intermediário e $27 \%$ no estrato inferior. Savia dictyocarpa foi a única que apresentou distribuição agregada em todos os estratos. Além desta espécie, apenas Croton floribundus apresentou distribuição agregada no estrato superior, não tendo, porém, indivíduos amostrados nos outros estratos. Actinostemon concolor, presente apenas nos dois estratos inferiores, apresentou forte agregação em ambos. Aspidosperma polyneuron e Metrodorea nigra apresentaram, ambas, distribuição aleatória no estrato superior e agregada nos estratos intermediário e inferior. A distribuição dos indivíduos de Actinostemon concepcionis foi regular nos estratos superior e intermediário, mas apresentou forte agregação no estrato inferior.

A cobertura das copas, estimada com base no somatório da projeção das copas das árvores sobre a área amostrada, foi, em média, de $179 \%$ para o estrato superior e $59 \%$ para o estrato intermediário, resultando em $238 \%$ de cobertura sobre o estrato inferior. A cobertura reflete o nível de sombreamento e valores superiores a $100 \%$ indicam superposição de copas.

Quanto maior a superposição de copas, menor a incidência de luz nos estratos inferiores e isso certamente dificulta o crescimento de lianas e a regeneração de espécies heliófitas, de estádios sucessionais iniciais. Nas parcelas em que a concentração de lianas foi muito alta (mais de 100 lianas/parcela), a cobertura das copas foi inferior a $100 \%$, possibilitando a incidência de luz solar direta em parte do piso florestal (figura 2). Em média, nesta área de amos-

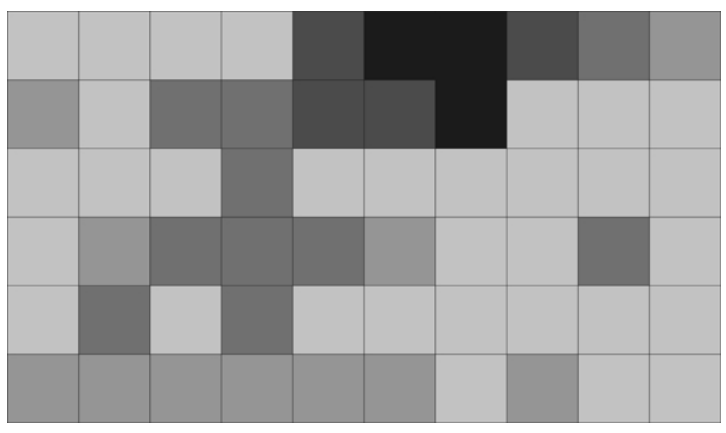

Figura 2. ( $\square$ menos de 10 lianas/parcela, cobertura 382\%; ( ) $\square$ a 20 lianas/parcela, cobertura 226\%; ( $\square 20$ a 50 lianas/ parcela, cobertura 178\%; ( $\square 50$ a 100 lianas/parcela, cobertura 125\%; ( 100 a 200 lianas/parcela, cobertura 93\%. 
tragem de floresta madura, ocorreram 28 lianas/parcela. Oito parcelas (13\% da área), nas quais ocorram quedas recentes de árvores, continham $65 \%$ das lianas, com densidade média de 138 lianas/parcela, enquanto que nas 52 parcelas restantes $(87 \%$ da área), onde não existiam clareiras, a média foi de 11 lianas/parcela.

$\mathrm{Na}$ análise global dos resultados obtidos, destaca-se a baixa diversidade alfa encontrada, em comparação com outras áreas de floresta estacional semidecidual, e a constatação de que grande parte das espécies que compõem o dossel não foram observadas em regeneração nesta área de floresta madura. A floresta madura, porém, constitui-se em apenas um dos componentes do mosaico florestal da Estação, descrito por Mattos et al. (1996), condicionado pelas variações no meio físico e pelos processos sucessionais. Na sua complexidade, a floresta como um todo pode abrigar um número bem maior de espécies e condições ecológicas adequadas para os processos de regeneração de cada uma delas.

\section{Referências bibliográficas}

BAITELLO, J.B., PASTORE, J.A., AGUIAR, O.T., SÉRIO, F.C. \& SILVA, C.E.F. 1988 . A vegetação arbórea do Parque Estadual do Morro do Diabo, município de Teodoro Sampaio, Estado de São Paulo. Acta Botanica Brasilica 1:221-230.

BERNACCI, L.C. \& LEITÃO-FILHO, H.F. 1996. Flora fanerogâmica da floresta da Fazenda São Vicente, Campinas, SP. Revista Brasileira de Botânica 19:149-164.

BRANNSTROM, C. 1998. After the forest: environment, labor and agrocommodity production in Southeastern Brazil. $\mathrm{PhD}$ dissertation, University of Wisconsin, Madison.

BROWN, W.H. 1919. Vegetation of the Philippine Mountains. Bureau of Science, Manilla, Publ. 13.

BRUCE, D. \& SCHUMACHER, F.X. 1950. Forest mensuration. McGraw-Hill Book Company, New York.

CARDOSO-LEITE, E. 1995. Ecologia de um fragmento florestal em São Roque, SP: florística, fitossociologia e silvigênese. Dissertação de mestrado, Campinas, Universidade Estadual de Campinas, SP.

CAVASSAN, O., CESAR, O. \& MARTINS, F.R. 1984 Fitossociologia da vegetação arbórea da Reserva Estadual de Bauru, Estado de São Paulo. Revista Brasileira de Botânica 7:91-106.

CERSÓSIMO, L.F. 1993. Variações espaciais e temporais no estabelecimento de plântulas em trechos de florestas secundárias em São Paulo, SP. Dissertação de mestrado, Universidade de São Paulo, São Paulo.
CESAR，O \& \& LEITÃO-FILHO, H.F. 1990. Estudo fitossociológico de mata mesófila semidecídua na Fazenda Barreiro Rico, Município de Anhembi, SP. Revista Brasileira de Biologia 50:443-452.

CHAPMAN, H.H. \& MEYER, W.H. 1949. Forest mensuration. McGraw-Hill Book Company, New York.

COSTA, L.G.S. \& MANTOVANI, W. 1992. Composição e estrutura de clareiras em mata mesófila semidecídua na Bacia de São Paulo. In II congresso nacional de essências nativas. São Paulo, SP. Revista do Instituto Florestal 1:178-183.

DEAN, W.1997. A ferro e fogo: a história da devastação da mata atlântica brasileira. Companhia das Letras, São Paulo.

FLOYD D.A. \& ANDERSON, J.E. 1987. A comparison of three methods for estimating plant cover. Journal of Ecology 75:221-228.

HALLÉ, F., OLDEMAN, R.A.A. \& TOMLINSON, P.B. 1978. Tropical trees and forests. An architetural analysis. Springer-Verlag, Berlin.

HUMBOLDT, F.H.A. 1808. Ansichten der natur mit wissenschaftlichen erläuterungen. Stuttgart \& Augsburg.

KRONKA, F., MATSUKUMA, C.K., NALON, M.A., DEL CALI, I.H., ROSSI, M., MATTOS, I.F.A., SHIN-IKE, M.S., PONTINHAS, A.A.S. 1993. Inventário florestal do Estado de São Paulo. Instituto florestal/SMA, São Paulo.

LUDWIG, J.A. \& REYNOLDS, J.F. 1988. Statistical ecology. A primer on methods and computing. J. Wiley \& Sons, New York.

MAGURRAN, A.E. 1988. Ecological diversity and its measurement. Princeton Univ. Press., Princeton.

MATTOS, I.F.A., ROSSI, M., SILVA, D.A. \& PFEIFER, R.M. 1996. Levantamento do meio biofísico e avaliação da fragilidade do ecossistema na Estação Ecológica dos Caetetus, SP. Sociedade e Natureza 15:388-393.

MMA, 1998. Primeiro relatório nacional para a Convenção sobre Diversidade Biológica: Brasil. Ministério do Meio Ambiente, dos Recursos Hídricos e da Amazônia Legal, Brasília.

MILDBRAED, J. 1922. Wissenschaftliche ergebnisse der zweiten deutschen Zentral-Afrika-expedition 1910-1 unter führung Adolf Friederichs, Herzogs zu Mecklenburg. Llinkhardt \& Biermann, Leipzig.

MULLER-DOMBOIS, D. \& ELLENBERG, H. 1974. Aims and methods of vegetation ecology. John Wiley \& Sons, New York.

NICOLINI-GABRIEL, E.M. \& PAGANO, S.N. 1992. Composição florística do estrato arbóreo de floresta mesófila semidecídua no município de Jahu, SP. Arquivos de Biologia e Tecnologia 35:725-748.

NICOLINI-GABRIEL, E.M. \& PAGANO, S.N. 1993. Estrutura fitossociológica do estrato arbóreo de floresta mesófila semidecídua no município de Jahu, SP. Arquivos de Biologia e Tecnologia 36:165-184.

PAYANDEH, B. 1970. Comparison of method for assessing spacial distribution of trees. Forest Science 16:312-317.

RICHARDS, P.W. 1996. The tropical rain forest: an ecological study. Cambridge University Press, Cambridge.

ROZZA, A.F. 1997. Florística, fitossociologia e caracterização sucessional em uma floresta estacional semidecidual: Mata da Virgínia, Matão, SP. Dissertação de mestrado, Universidade Estadual de Campinas, Campinas. 
SCHLITTLER, F.H.M., MARINIS, G. \& CESAR, O. 1995. Estudos fitossociológicos na floresta do Morro do Diabo (Pontal do Paranapanema, SP). Arquivos de Biologia e Tecnologia 38:217-34.

SHEPHERD, G.J. 1994. FITOPAC 1. Manual do usuário. Departamento de Botânica, UNICAMP, Campinas.

SILVA-FILHO, S.R. \& ENGEL, V.L. 1993. Estrutura de um fragmento de mata mesófila semidecídua secundária tardia e implicações para o manejo. In Anais do $1^{\underline{0}}$ congresso florestal panamericano e $7^{\underline{0}}$ congresso florestal brasileiro, SBS, Curitiba, p.343-352.

SMA 1998. Atlas das unidades de conservação ambiental do Estado de São Paulo. Parte II. Interior. Secretaria de Estado do Meio Ambiente, Metalivros, São Paulo.

STRANGUETTI, V. 1996. Levantamento florístico das espécies vasculares de uma floresta estacional no norte do Estado de São Paulo, Estação Ecológica de Paulo de Faria. Tese de doutorado, Universidade Estadual de Campinas, Campinas.
TABANEZ, A.A.J., VIANA, V.M. \& NASCIMENTO, H.E.M 1997. Controle de cipós ajuda a salvar fragmentos de floresta. Ciência Hoje 22:58-61.

TABARELLI, M. \& MANTOVANI, W. 1997. Colonização de clareiras naturais na floresta atlântica no sudeste do Brasil. Revista Brasileira de Botânica 20:57-66.

VELOSO, H.P., RANGEL FILHO, A.L.R. \& LIMA, J.C.A. 1991. Classificação da vegetação brasileira, adaptada a um sistema universal. Fundação Instituto Brasileiro de Geografia e Estatística, Rio de Janeiro.

ZICKEL, C.S. 1995. Fitossociologia e dinâmica do estrato herbáceo de dois fragmentos florestais do Estado de São Paulo. Tese de doutorado. Universidade Estadual de Campinas, Campinas. 